\title{
An Dynamic Hello Messaging Scheme for Reducing Energy Consumption in On-Demand MANET Routing Protocols
}

\author{
Abhishek Nadda \\ Department of Computer Science Engineering \\ Indo Global college of Engineering, Abhipur \\ Mohali, India
}

\author{
Under the guidance of Pooja Wadhwa \\ Department of Computer Science Engineering \\ Indo Global college of Engineering, Abhipur \\ Mohali, India
}

\begin{abstract}
The increase in availability and popularity of mobile wireless devices has lead researchers to develop a wide variety of Mobile Ad-hoc NET working (MANET) protocol so exploit the unique communication opportunities presented by these devices. In mobile ad-hoc networks using smart phones energy efficiency is a major issue and for the local link connectivity information used in neighbor discovery, periodically exchanging hello messages are preferred. Due to neighbor discovery messages high overhead is produced in the On-Demand routing protocols, such as in AODV. In an attempt to enhance the scheme, this paper proposes a new approach to solve the problem by reducing unnecessary hello messages. In this paper we consider MANET under random waypoint model and investigated the relationship between the hello interval and event intervals. Finally we evaluate our study through simulations, the proposed scheme reduces the energy consumption and overhead of neighbor discovery processes.
\end{abstract}

Index Terms - Hello messaging, energy consumption, network overhead, hello interval, mobile ad-hoc network (MANET).

\section{INTRODUCTION}

A Mobile ad-hoc network is a multi-hop self-configuring network where mobile nodes communicate with other nodes directly or indirectly over wireless links without any preinstalled infrastructure. Nodes act as hosts as well as routers [1]. The MANET topology changes very frequently since the nodes are capable to move and due to this we need to cope with the problems raised [2]. Due to the highly dynamic nature of the Manet, routing is the key issue. In Manet all the nodes are capable of movement and connected to each other dynamically but they are vulnerable to failure. Since the mobile nodes are powered by the batteries the limited capacity and energy efficiency are one of the important criteria for Manet. Failure of mobile node due to power not only affects the node itself but also its ability to forward the packets and also it affects the lifetime of the network. So routing of packets in such an environment is difficult, however number of protocols have been developed for MANET [1]. Two commonly used routing strategies are reactive and proactive. Reactive routing protocols creates routes only when they are required (e.g., DSR, AODV). Proactive routing protocols are table driven and utilize table to determine the next hop to reach the destination (DSDV, OLSR, TORA). The major concern in a mobile-adhoc network using smart phones is the energy efficiency. Discovery of neighbor nodes in a Manet can be a hidden energy drain due to no-sleep energy bugs in Manet. In Manets, any node in a route can be turned off or can move away, which negatively affects route maintenance and may cause delay in the data dissemination. It is important for a node in Manet to discover live neighbor nodes through hello messaging [3]. Hello messages must be generated and transmitted at each Manet node independently. Two successive transmissions of hello message on the same MANET node must be separated by least hello interval [4].

In conventional hello messaging scheme, the hello messages are sent on the basis of hello interval that should be kept constant for hello messages broadcasting to the network for route establishment and maintenance [5] and also no start/end condition is described [3]. Most ad hoc routing protocols may detect broken links using hello messages and the feedback provided to the protocol by MAC (Medium Access Control) layer. At low network load MAC feedback works better than hello messages but when the traffic load on the network increases then due to incorrect link failure decisions throughput decreases. Routing protocols require hello messaging when run over the link layer protocols that do not provide MAC feedback when the next hop is unreachable. In standard AODV using periodic hello messaging, failed links are to be detected before a packet is sent to the next hop. But MAC layer protocols do not provide feedback about the presence of the next hop. Also the consumed energy and the volume of the control traffic is greater when AODV is run over MAC layer protocols [6]. Hence hello messaging is preferred over Link layer feedback mechanism. Giruka and Singhal proposed two approaches for suppressing the hello messages whenever they are not required, an on-demand mechanism and a monitoring activity mechanism. The ondemand mechanism also called hello protocol enables hello protocol only when it is demanded using a hello request-reply mechanism, but because of additional packet exchange before communication delay increases. The monitoring activity mechanism also called event based hello protocol and it enables only those nodes to broadcast hello packets that are active, the hello packets are broadcasted based on a threshold called an activity timer [7].

In this paper we investigate the dynamic hello messaging scheme for neighbor discovery, which belongs to the ondemand Manet routing protocols. The dynamic hello messaging scheme aims to reduce the unnecessary hello messages by making hello interval proportional to the event 
interval of a node. First we design the network by taking random network topology and random events are taken and hello messaging scheme used initially by using constant hello interval. Then we enhance the scheme by making hello interval proportional to event interval i.e. as the event will take place the node will start sending hello messages, hence hello messaging scheme made dynamic. Via simulation experiments we evaluate these schemes under different parameter settings of dynamic environment to see their performance in MANETs related to energy efficiency and battery consumption.

The rest of paper is organized as follows: In section II related work is discussed. The proposed method of dynamic hello messaging scheme is presented in section III. In section IV we give the results obtained by applying method to the MANET. Finally section V concludes this paper with some discussions on the future work.

\section{RELATED WORK}

The hello messaging scheme has been investigated extensively in the literature. In [8], an implementation of Ad hoc on demand distance vector (AODV) is utilized to determine the effectiveness of hello messages for determining local connectivity. To increase the effectiveness of the hello messages, the reception characteristics of hello messages should be equal to that of the data packets and for this two must have similar characteristics of rate and size and hence better throughput will result in this case. In [6], the advantages and drawbacks of both the techniques hello messaging and MAC feedback i.e. used to determine link failures in ad hoc routing protocols are addressed. Simulation results showed that MAC feedback works better than hello messages with low network load, but if the network load increases, the incorrect decision about link failure also increases which results in lower throughput. Also when AODV is run over MAC layer protocols, the energy that is consumed and traffic control consumed with the MAC protocols are greater than when they run over MAC IEEE 802.11. In [7], impact of the hello protocols on adhoc networks is discussed. The basic idea behind the proposed scheme is to send hello messages as little as possible, the proposed hello protocols incur less overhead and the network performance increases compared to a periodic hello messaging protocol, while maintaining the accuracy of identical neighbor table. In [9], the relationship between the transmission frequency of hello messages and the sensing timer expiration value is investigated with the network's node mobility. In [10], neighbor discovery is exploited and overhead of neighbor discovery processes is reduced.

However all these schemes address only conventional hello messaging scheme using constant hello interval. But the network topology changes rapidly in MANETs due to random node mobility and for neighbor discovery hello messages are sent after regular intervals i.e. constant hello interval and hence battery consumption increases, energy efficiency decreases.

\section{PROPOSED SCHEME}

In this section we first present our network model and then formulate the hello messaging scheme. We consider MANET operating within random network topology and random nodes. Node mobility follows a model, there is several mobility models used in the evaluation of MANET protocols. The random waypoint model is one the most commonly used mobility models for simulations of MANETs. In this model, each node selects a random destination, uniformly distributed within the two-dimensional space [11]. In conventional hello messaging scheme before a packet is sent, status of neighbor nodes should be recognized first so as to recognize if there is a link failure with one of its neighboring nodes. If the node moves to an area where no active nodes are in its neighborhood, then nodes keeps broadcasting hello messages and due to this unnecessarily energy consumption takes place. For this problem hello messages should be suppressed first on the basis of determining value of hello interval. The maximum interval of time between the transmissions of hello messages is HELLO_INTERVAL. The time during which the node should assume that link is currently broken is the time if a node does not receive any packet from that node within the given time i.e.

$\mathrm{T}_{\mathrm{FD}}=($ ALLOWED_HELLO_LOSS $) *($ HELLO_INTERVAL). (1)

By default, ALLOWED_HELLO_LOSS is equal to 2 and HELLO_INTERVAL is equal to $1000 \mathrm{~ms}$ i.e. $1 \mathrm{sec}$ for AODV [12]. Now in the dynamic hello messaging scheme the time for link failure detection $\left(\mathrm{T}_{\mathrm{FDD}}\right)$ is given as:

$\mathrm{T}_{\mathrm{FDD}} \quad$ (ALLOWED_HELLO_LOSS $-\quad 0.5$ ) *(HELLO_INTERVAL).

(2)

The main objective of our research is to reduce energy consumption and network overhead. This can be done only when hello interval is made proportional to the event interval of a node. If the hello interval is set without considering the event interval of a node, then the risk of sending packet through an unavailable link increases $\left(\mathrm{P}_{\mathrm{FDD}}\right)$. In our research we are considering the scenario of an urban city and Exponential traffic distribution for our research. We assumed the $\mathrm{C}_{\mathrm{DF}}$ of event interval in Fig. 1 and the assumption is considered for the 100 events, where it is analyzed for the time of 1 hour and is given by:

$$
\mathrm{X}(\mathrm{a}, \mathrm{b})=1-\mathrm{e}^{-\mathrm{a} / \mathrm{b}}
$$

We consider $X(a, b)$ as probability of the total no of outcomes bounded by exponential distribution. We can write this as:

$$
\begin{aligned}
& P_{F D D}=1-e^{-a / b} \\
& \ln \left(P_{F D D}\right)=\ln \left(1-e^{-a / b}\right) \\
& \ln \left(P_{F D D}\right)=\ln (1)-\ln \left(\mathrm{e}^{-a / b}\right) \\
& a=-b \ln \left(1-P_{F D D}\right)
\end{aligned}
$$

Here $a$, is in linear relationship with $b$, so the neighboring node to calculate the value of hello interval use $b$, to maximize the value of $\mathrm{T}_{\mathrm{FDD}}$. With the value of $\mathrm{T}_{\mathrm{FDD}}$, we can calculate the value of hello interval by using equation 2 .

The distribution of the packet reception of the event intervals on each node is investigated with 20 nodes. The most of event intervals are less than the default hello interval i.e. $1 \mathrm{sec}$; we 
investigated the event intervals that are larger than the $1 \mathrm{sec}$, because hello interval will not be modified if value will be less than $1 \mathrm{sec}$. The probability of failure detection of an unavailable link is made proportional to exponential traffic distribution.

\section{EVALUATION}

The network size was fixed to 20 nodes located in a $1000 \mathrm{mx} 1000 \mathrm{~m}$ square. A random way point model is chosen as the mobility model, in this model nodes choses a random destination point. We concentrated on two performance metrics in the simulation: network overhead and energy consumption.

We modified AODV and TORA with the proposed scheme i.e. AODV-DY and TORA-DY respectively. The default Pfd is set to $20 \%$. The simulation parameters shown in Table I are used.

Fig. 2 compares energy consumption between TORA and TORA-DY. Each node has initially 1 joule of energy. As time increases TORA consumes energy quickly due to periodic hello messaging scheme and hence more energy consumption and battery drain will take place. When TORA-DY is applied, hello messages are suppressed and hence energy consumed is less due to less transmissions and reception of hello messages. Fig. 3 shows the hello packet overhead. The number of the hello packets is decreased by as much as half due to the proposed scheme, because as no of nodes will increase, the number of hello packets received by a node will also increase. Fig. 4 compares the energy consumed by AODV and AODVDY. Each node has initially 160 joules of energy. Here remaining average energy of all nodes are taken. As number of nodes will increase AODV consumes energy quickly, while AODV-DY due to the proposed scheme will reduce unnecessary hello messages hence, take more time for energy consumption.

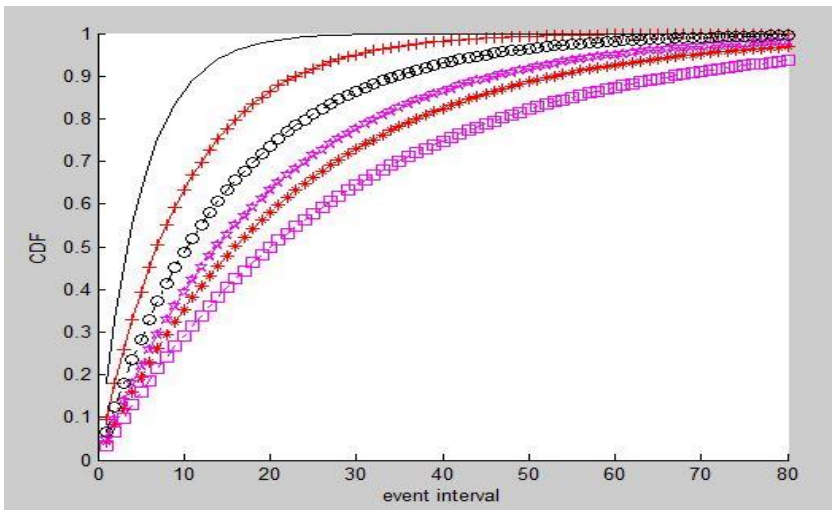

Fig.1. Distribution of event intervals

\begin{tabular}{|c|c|}
\hline Parameter & Value \\
\hline Mobility model & Random way point model \\
\hline $\begin{array}{c}\text { Transmission } \\
\text { power }\end{array}$ & $16 \mathrm{dBm}$ \\
\hline Topology size & $1000 \mathrm{mx} 1000 \mathrm{~m}$ \\
\hline No of nodes & 20 \\
\hline
\end{tabular}

\begin{tabular}{|c|c|}
\hline Packet size & 210 bytes \\
\hline Simulation time & 30 \\
\hline Data traffic & Exponential \\
\hline Pause time & $0 \mathrm{sec}$ \\
\hline
\end{tabular}

TABLE I. Simulation Parameters

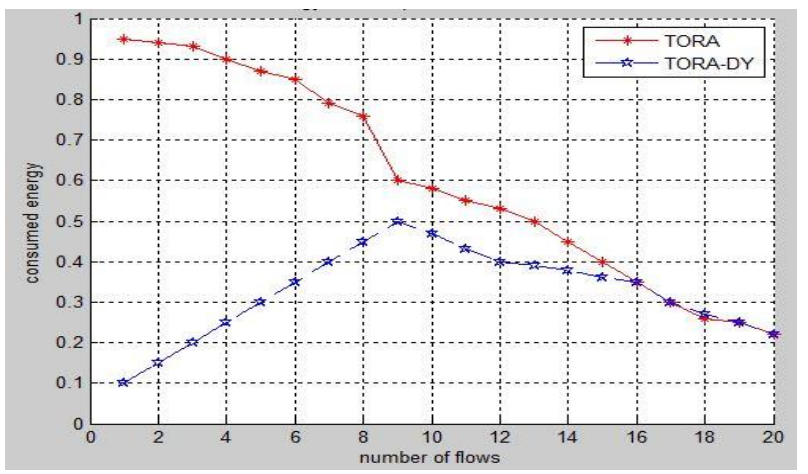

Fig.2. Energy consumption for variable flows

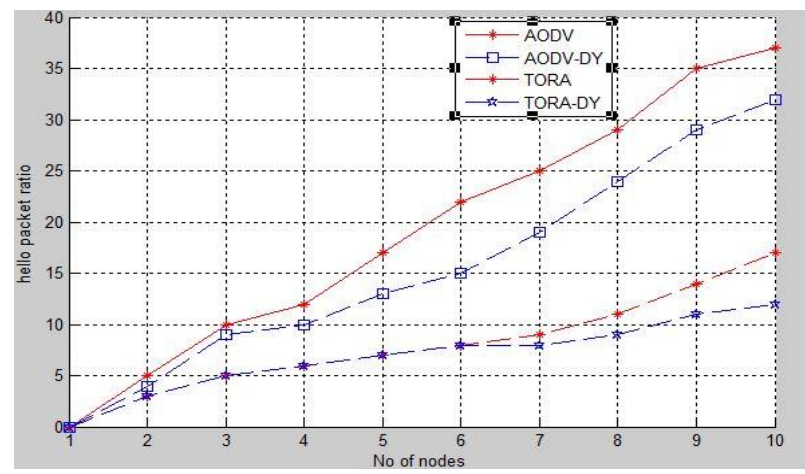

Fig.3. Hello packet overhead

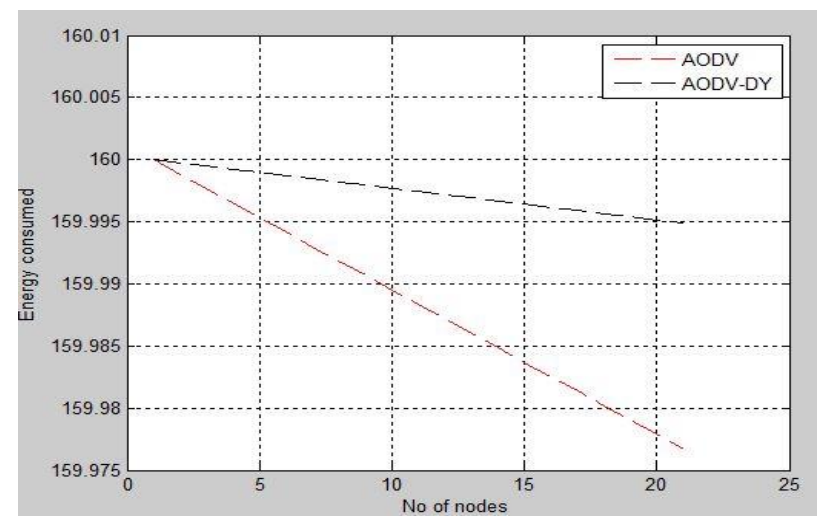

Fig.4. Energy consumption for AODV

\section{CONCLUSION AND FUTURE WORK}

In this paper, a new approach, called Dynamic Hello Messaging scheme is proposed to solve the problems related to battery consumption and network overhead. The Hello Messaging Scheme aims to reduce unnecessary hello messages while neighbor discovery and also to establish a 
reliable connection between the source node to the destination node. This is one of the important issues that significantly affect the performance of the MANETs.

As a future work, we can deploy the proposed approach in more scenarios and large scale networks, and we plan to extend our work to optimize the value of the hello intervals by using different optimization techniques.

\section{REFERENCES}

I. Chlamtac, M. Conti, and J. J. Liu. Mobile ad hoc networking: Imperatives and challenges. Ad Hoc Networks, 1(1):13-64, 2003. Cited By (since 1996):376.

2. S.Corson, J. Macker., "Mobile Ad hoc Networking (MANET): Routing Protocol Performance Issues and Evaluation Considerations," IETF RFC2501, 1999.

3. Seon Yeong Han, and Dongman Lee "An Adaptive Hello Messaging Scheme for Neighbor Discovery in On-Demand MANET Routing Protocols" IEEE Communications Letters, VOL. 17, NO. 5, MAY 2013.

4. T.Clausen, C. Dearlove, and J. Dean, "Mobile ad hoc network (MANET) neighborhood discovery protocol (NHDP)," 2010.

5. David B. Johnson, David A. Maltz, Yih-Chun Hu and Jorjeta G. Jetcheva, "The Dynamic Source Routing for Mobile Ad Hoc Wireless Networks, “ July 2004.

6. Dimitri Marandin, "Performance Evaluation of Failed Link Detection in Mobile Ad Hoc Networks," 3rd Annual Med-HocNet, 2004.

7. V.C. Giruka and M. Singhal, "Hello protocols for ad-hoc networks: overhead and accuracy tradeoffs," in Proc. Sixth IEEE International Symposium on a World of Wireless Mobile and Multimedia Networks, pp. 354-361.

8. Ian D.Chakeres, Elizabeth M. Belding-Royer, "The Utility of Hello Messages for Determining Link Connectivity" in Proc. 2002 Wireless Personal Multimedia Communications, 2002. The 5th International Symposium.

9. R. Oliveira, M. Luis, L. Bernardo, R. Dinis, and P. Pinto, "The impact of node's mobility on link-detection based on routing hello messages," in Proc. 2010 IEEE Wireless Communications and Networking Conference, pp. 1-6.

10. V. D. Tracy Camp, Jeff Boleng, "A survey of mobility models for ad hoc network research," Wireless Communications and Mobile Computing, 2:483-502, 2002.

11. E. Belding-Royer and S. D. C. Perkins, "Ad hoc on-demand distance vector (AODV) routing,” July 2003. 